\title{
The Role of (In)Visibility in Hate Crime Targeting Transgender People
}

\author{
Dr Ben Colliver \\ Birmingham City University \\ School of Social Sciences \\ Curzon Building \\ 4 Cardigan Street \\ B4 7BD \\ Ben.colliver@bcu.ac.uk \\ 01213316234
}

\section{Dr Marisa Silvestri}

University of Kent

School of Sociology, Social Policy and Social Research

Cornwallis East

Canterbury

Kent

CT2 7NZ

M.silvestri@kent.ac.uk

01227827437

Ben Colliver is a Lecturer in Criminology at Birmingham City University. His research interests include hate crime, gender and sexuality. His research focuses on discrimination and hate crime targeting transgender and non-binary communities. He is a member of the steering group of the British Society of Criminology Hate Crime Network.

Marisa Silvestri is Reader in Criminology at the University of Kent. Her work lies at the intersections of gender, crime and criminal justice. She has a longstanding record of research and has published extensively in the field.

Word Count: 9,335 including references 


\section{Abstract}

This paper explores incidents of hate crime targeted at transgender people. Drawing on in-depth interview data, it challenges and extends established theorisations of the significance of 'difference' and 'vulnerability' in relation to victimisation. It introduces and emphasises the idea of 'visibility' as a more useful lens through which to understand the systematic harm experienced by hate crime victimisation. Through an analysis of complex identities, the paper argues that current conceptualisations of 'visibility' do not appreciate the complex, intersectional nature of visibility, which is key in understanding how people navigate their identities in different spaces and contexts.

Keywords: Visibility, Transgender, Hate Crime, Difference, Vulnerability 


\section{The Role of (In)Visibility in Hate Crime Targeting Transgender People}

\section{Introduction}

The term 'hate crime' has recently garnered significant attention in social, political and academic spheres and is now arguably embedded within a number of academic disciplines (Chakraborti and Garland, 2012). Encompassing a broad range of victimisation, hate crimes are a subset of crimes representing around 1$2 \%$ of recorded crime in England and Wales (Home Office, 2019). Although a small percentage of overall crime, incidents of recorded hate crime are increasing annually with 103,379 hate crimes being recorded by police forces in 2018-19, an increase of $10 \%$ from the previous year (Home Office, 2019).

Hate crimes targeting an individual's transgender identity account for the smallest amount of recorded hate crime, standing at $2 \%$ (Home Office, 2019), however, the total number of transphobic hate crimes has increased year on year since it was added to the list of monitored categories in 2009. It is likely that official statistics significantly underestimate the prevalence and extent of transphobic hate crime, and studies have shown significantly higher rates of victimisation (Chakraborti et al., 2014; METRO, 2014). High rates of victimisation targeting transgender people have been documented in studies across the global north (Antjoule, 2013; James et al., 2016; Turner et al., 2009). 
In this paper, we focus our attention on transphobic hate crime, that is, incidents of hate crime targeted at transgender individuals. We draw on Hines's (2010) definition of 'transgender' in which she notes transgender as:

'a range of gender experiences, subjectivities and presentations that fall across between or beyond stable categories of 'man' and 'woman'. Transgender includes gender identities that have, more traditionally, been described as 'transsexual', and a diversity of genders that call into question an assumed relationship between gender identity and presentation and the 'sexed' body'. (Hines, 2010:1)

This definition of 'transgender' is particularly useful as it acknowledges gender expressions that fall between and beyond the gender binary of 'man' and 'woman'. We appreciate, however, that there is some contention within and between communities over the use of the term 'transgender' and Monro (2003) acknowledges the problematic nature of the term, suggesting that the inclusivity of such an array of social groupings neglects to acknowledge their range of needs and interests. The abbreviated term 'trans' is also used throughout this paper to denote transgender identities. When the term 'trans' is used, it can be assumed to function as an umbrella term, inclusive of all people who do not 
identify as cisgender ${ }^{1}$. Despite the inclusivity of gender identities within this research, it is important to note that there is no specific legal recognition, or protection of non-binary identities in the United Kingdom (UK).

Made up of three parts, the first part of the paper explores official and academic conceptualisations of hate crime and draws attention to some of the problematic definitional issues that have shaped the discourse on hate crime. We go on to outline the methodological approach and processes adopted in the study. In the third and final part, we report our findings and emphasise the concept of 'visibility' through two key themes developed from the interview data, that of, 'intersectional visibility' and 'discursively constructed visibility'. In this final section, our three key arguments are developed. Firstly, that existing dichotomous concepts of (in)visibility are limited, in that they may only be useful in understanding some identities at particular times and in specific spaces and therefore do not consider the complicated nature of (in)visibility. In this paper, we emphasise greater complexity through an appreciation of the ongoing negotiation and fluidity of identities. We draw upon aspects of intersectionality throughout to demonstrate the complexity in negotiating (in)visibility, emphasising the significance of intersecting social identities and the implications of occupying

\footnotetext{
${ }^{1}$ This term is used to refer to those who identify with the gender they were assigned at birth, based on observation of biological sex.
} 
multiple marginalised social positions (Mattias de Vries, 2012). The analysis presented here affords a more nuanced reading of 'visibility' in which both the obvious tangible qualities and the more imperceptible discursive experiences of visibility are present and acknowledged.

Secondly, through an appreciation of the intersectional nature of visibility, we argue that 'visibility' operates in complex ways: both as a facilitator of hate crime and as a preventative barrier to victimisation. In this sense, we demonstrate that increased visibility does not necessarily equate to an increased risk of victimisation, or perceived 'vulnerability'. Instead, in particular cases, a heightened level of visibility may in fact decrease an individuals' sense of vulnerability to victimisation. In developing our understanding of '(in)visibility' beyond the existing dichotomy, we explore the ways in which trans identities are navigated in particular spaces and contexts.

Finally, we argue that hate crime victimisation should be explored through the lens of '(in)visibility', alongside dominant notions of 'difference' and 'vulnerability'. This will allow for a deeper, more critical appreciation of the experiences, and social contexts within which hate crimes occur. These contributions are significant to criminological thought, in advancing our understanding of the contextual and spatial challenges of navigating personal identities. 
With the analysis of transphobic hate crime very much in its infancy both in the UK and beyond, our findings, analysis and arguments have international reach and significance. Whilst the empirical data presented in this paper are firmly grounded within a UK context, the conceptual contributions that we make can be applied internationally. Throughout this paper we use the term '(in)visibility' to signify the dichotomous conceptualisation of both the increasing and decreasing nature of visibility, dependent upon the particular social context within which it is operating. We do, however, refer directly of visibility, and invisibility, when discussing instances or contexts which lead specifically to an increase or decrease in visibility.

Drawing upon 31 in-depth interviews with trans people, we provide empirical data on their experiences of transphobic hate crime. The data used in this article were collected as part of a larger research project that sought to identify the lived experience of trans people who experience less socially recognisable forms of hate crime targeting trans people, such as verbal abuse and harassment that fall under the Public Order Act (1986). Despite this focus discussions regularly centred on incidents of physical and sexual violence. Given the diverse and representative nature of our sample in comparison to the UK population, in relation to gender identity, ethnicity, religion, age and disability status (see methodology below), we explore the impacts of intersectionality on 
trans people's experiences of hate crime. In so doing, we provide new insights and data on a hard to reach, vulnerable and under-researched group. We extend dominant theoretical frameworks of hate crime victimisation that have focused on 'difference' (Perry, 2001) and 'vulnerability' (Chakraborti and Garland, 2012) as key aspects in understanding hate crime and propose the concept of 'visibility' as a more useful lens through which to make sense of the complex nature of identities at play in incidents of transphobic hate crime.

\section{Conceptualising Hate Crime and (In)Visibility}

Despite the increased awareness and acknowledgment of the concept of hate crime, there is no universally agreed definition of what a hate crime actually is. Moreover, there are significant differences between academic and legal definitions, making investigation and analysis difficult. Legal definitions focus solely on an individual, isolated incident of criminality (thereby reinforcing perceptions of hate crimes as single, extreme, public incidents). Academic definitions, by contrast, tend to focus on wider social, cultural and political structures that create a climate in which particular groups of people are deemed legitimate targets for hate crime (Perry, 2001). Although there is no statutory definition of hate crime in the UK, the term hate crime has been defined by The Home Office (2012) as: 
'any criminal offence which is perceived, by the victim or any other person, to be motivated by a hostility or prejudice based on a personal characteristic.' (2012:6)

The personal characteristics referred to here, which require annual monitoring by police forces, include race, religion, disability status, sexual orientation and trans identity (Home Office, 2012). The Legal Aid, Sentencing and Punishment of Offenders Act (2012) amended section 146 of the Criminal Justice Act (2003) to include trans identity as a characteristic to be considered as an aggravating factor during the sentencing of an offender. Not only did this legislation define trans identity as an aggravating factor, it further increased the starting punishment from 15 years imprisonment to 30 years imprisonment in relation to transphobically aggravated murder. Similar legislative provisions have been introduced in Scotland in the form of the 'Offences (Aggravated by Prejudice) Scotland Act in 2009. This act established 'transgender identity' as a protected characteristic. Scotland's approach offers more progressive legislative protection than England and Wales by giving legal recognition to non-binary identities. At the time of writing, there is currently no specific legal protection for trans people in Northern Ireland. However, an independent review of hate crime legislation is currently being undertaken in Northern Ireland to review issues relating to definitions and protected characteristics. 
The problematic nature of official definitions of hate crime has been well rehearsed by a number of commentators (see for example, Gerstenfeld, 2004; Hall, 2005; Jacobs and Potter, 1998). The subjective nature of legal definitions has also been acknowledged within academia alongside the over-focus on isolated incidents, rather than an appreciation of the wider socio-political climate which culminate in incidents of hate (Perry, 2001). In academic discourses, it is Perry's (2001) definition of hate crime that has emerged as key, noting that:

'Hate crime ... involves acts of violence and intimidation, usually directed towards already stigmatised and marginalised groups. As such, it is a mechanism of power and oppression, intended to reaffirm the precarious hierarchies that characterise a given social order. It attempts to re-create simultaneously the threatened (real or imagined) hegemony of the perpetrator's group and the 'appropriate' subordinate identity of the victim's group. It is a means of marking both the Self and the Other in such a way as to re-establish their 'proper' relative positions, as given and reproduced by broader ideologies and patterns of social and political inequality.' (2001:10)

Perry claims that hate crime is better understood as an extreme form of discrimination against those already ostracised by society as 'different', which is stimulated by a culture of othering and segregation. Here, 'difference' is socially 
constructed in negative relational terms (Perry, 2001:47). The 'difference' that Perry speaks of relates to a number of different social hierarchies pertaining to gender, sexuality, race and disability among other identity markers. Through the construction of these social hierarchies, a range of 'in-groups' and 'out-groups' are constructed - Cisgender is established as the dominant norm, in which all others are (unfavourably) judged. As the dominant norm, cisgender identities rarely encounter the same level of interrogation that trans identities do. Bauer et al. (2009:356) define 'cisnormativity' as the 'expectation that all people are cissexual, that those assigned male at birth always grow up to be men and those assigned female at birth always grow up to be women'. Those who do not conform to this expectation, may be visibly marked as 'different'.

The 'difference' presented by the 'out-group' may lead to feelings of fear and insecurity within the 'in-group' about their dominant place in society. The dominant group must ensure that subordinate groups remain subordinate in order to maintain the relational power dynamics which Perry (2001:2) argues 'leave minority members vulnerable to systemic violence'. This power dynamic is maintained through the social policing of various minority groups which may manifest itself in animosity, discrimination and violence.

As an 'out-group' trans individuals experience violence as a result of complex social structures and hierarchies, as Jauk (2013:808) argues, 'violence 
against trans people is often triggered by gender non-conformity and violence is a form of gender policing'. When a transphobic hate crime is committed, a message reaffirming the trans communities' subordination to cisgender communities is conveyed, continuing the oppression that trans communities experience (Burgess et al., 2013). This has been conceptualised by Doan (2010:635) as the 'tyranny of gender'. The tyranny of gender occurs when individuals dare to challenge the expected social norms of gender. This may result in 'genderism' (Browne, 2004) which describes the hostile reading of gender ambiguous bodies. In the context of transphobic hate crime, violence results from a discontinuity between an individuals' gender presentation and assumptions made by others about an individuals' gender.

It can therefore be argued that trans individuals experience hate crime as an instrument of 'intimidation and control exercised' by those who need to reaffirm their place in an unfixed hierarchy (Perry, 2001:2). This claim is supported by academics who suggest that trans individuals who fail to present themselves according to society's accepted beliefs about male and female presentation and performance will be more at risk of experiencing 'regular and extreme levels of physical and verbal abuse' (Johnson et al., 2007:18; Spalek, 2008). It is important to acknowledge that both trans and cisgender communities do not exist as 
singular, cohesive groups, and both communities are diverse and differ in needs and desires (Jamel, 2018).

More recently, Chakraborti and Garland (2012) have offered a critique of the ways in which Perry's theory of 'doing difference' perpetuates the notion of 'stranger danger'. Stranger danger is the concept that victimisation happens primarily in public, perpetrated by an individual who is unknown to the victim. In reconceptualising conventional frameworks, they draw upon the work of McGhee (2007) to point to the spontaneity of many incidents of hate. Here, not all perpetrators of hate crimes are prejudiced all of the time but may act in a prejudicial or hateful way as the result of a particular 'trigger' event. It is therefore argued that the vast array of incidents may only be partly motivated by prejudice, thus challenging the assumption that the sole purpose of hate crimes is to act as a mechanism of oppression of the 'other'. In this reconceptualization, Chakraborti and Garland draw attention to groups of victims who are often marginalized in conventional frameworks, including, but not exclusively, homeless people and sex workers. They challenge the inevitability of encountering hate crime and suggest that a conceptual focus on 'vulnerability' better encapsulates offenders' perceptions of victims. It is argued that hate crime victimisation cannot be explained by an explicit focus on 'difference'. Rather, it is the victim's perceived vulnerability alongside their 'difference' that makes someone a target for hate 
crime victimisation. Chakraborti and Garland further argue that exploring hate crime victimisation through the lens of vulnerability allows for a more nuanced understanding of the interplay between recognised minority characteristics and wider social, class and political marginalisation. This is important to acknowledge as existing literature suggests that the likelihood of victimisation is dependent upon a number of factors including an individual's ability to 'pass' (Jamel, 2018) and the gender they present as (Kidd and Witten, 2008). In this paper, we extend these frameworks by considering how 'difference' and 'vulnerability' are conceptualised through the lens of 'visibility'.

Notions of (in)visibility have been applied in hate crime literature to varying degrees of detail and at various conceptual levels, from individual visibility to wider structural visibility (Mills, 2019; Perry, 2015; Wallengren and Mellgren, 2015). These studies have shown that greater levels of visibility of 'difference' result in higher levels of fear of victimisation. Notions of '(in)visibility' within existing hate crime literature have largely been explored in relation to Islamophobia (Chakraborti and Zempi, 2012; Perry, 2015). Perry (2015) discusses the visibility of Muslim communities and identities in a wider structural, political and mediatised contemporary society. She argues that post 9/11 Muslim identities became more visible due to the specific discourse around terrorism. Whilst this is particularly useful in conceptualising visibility in relation to wider 
structural systems of oppressions, it tends to overlook the role of visibility in relation to personal, individual identities on a micro-scale. Chakraborti and Zempi (2012) have conceptualised notions of 'visibility' in relation to the veil. Constructed as a significant visual indication of a Muslim identity, the process of 'veiling' becomes a visual indicator of 'difference'. Such commentaries of 'visibility' are however, focused heavily on the public nature of hate crime victimisation that fits within dominant frameworks that relate to 'stranger danger. The concept of 'stranger danger' is firmly established within hate crime literature (Gerstenfeld, 2004; Lawrence, 1999). However, others have suggested that victims of hate crime are likely to have a pre-existing relationship with the perpetrator (James et al., 2016; Mason-Bish, 2010; Meyer, 2014). Walters (2011:319) claims that 'cultures of prejudice are nurtured within families, friendship circles and by neighbours' and it would therefore be surprising to find that perpetrators of hate crime only victimise strangers.

Perry and Dyck (2014) provide one of the few studies that adopt a 'transcentred' approach to visibility. However, this is largely done in relation within the context of the 'pass-not pass' dichotomy and focuses primarily on public visibility, rather than any engagement with the dynamics of victimisation that may take place in the private sphere, including within the context of romantic and sexual relationships. By drawing attention to the blurred nature of (in)visibility and its 
relationship to public and private spaces, we attend to this oversight in this paper. The focus on 'stranger danger' constructs hate crime as a public issue, and neglects to acknowledge hate crimes that occur within the home. Stotzer (2009) discovered a significant portion of transphobic hate crime victims experienced these within the home, perpetrated by family members and friends. These experiences are often overlooked conceptually. It is also important to note that the public/private divide is not a straight-forward distinction and boundaries of private spaces may become even more blurred in the case of public toilets, locker rooms and other potentially shared spaces (Davidoff, 2003). That said, the image

of the 'stranger' is firmly located within the 'public sphere', contributing further to the overshadowing of violence perpetrated by those known to the victim.

\section{Visibility, 'Passing' and Queer Criminology}

In developing a greater understanding of 'visibility', it is important to acknowledge existing work that has considered this concept. Whilst the issue of visibility has been debated within hate crime scholarship, the complexity of the concept has not yet been fully realised nor gained significant prominence. It has, however, been considered more significantly within trans and queer scholarship (Kilian, 2014; Lovelock, 2017; Rundall and Vecchietti, 2010). Research here has explored the connection between visibility and 'passing'. What it means to 'pass' and the implications of 'passing' is an area of contention within trans literature, 
particularly at a time when gender identity is becoming increasingly politicised. Passing may be seen as living authentically, as a method for survival or may also be seen in more socially political terms as conforming to, and reinforcing rigid gender binaries (Roen, 2002). As such, 'passing' is often presented as a dichotomous concept, in which one either passes, or does not. However, scholars have critiqued this concept and identified that the idea of 'passing' is not a simple one (Serano, 2007). People may or may not pass, with, or without intending to, in different spaces and at different times. In this paper, we are concerned with the ways in which visibility interacts with the concept of 'passing' to provide a more nuanced debate of how this impacts trans people's experiences of hate crime.

Despite Queer criminology's relatively recent emergence (Ball, 2019), there has been a growing body of work that seeks to centre the lives and experiences of those whose identities may fall under the 'Queer' umbrella. This work seeks to challenge, interrogate and deconstruct dominant frameworks within which queer identities are produced and exist (Ball et al., 2014; Panfil, 2017; Woods, 2014). Our paper adopts this approach by centring trans people's experiences, an approach that within UK focused hate crime literature has often been overlooked. Indeed, three of the largest UK hate crime projects; Youth Chances, All Wales Hate Crime Project and The Leicester Hate Crime Project, 
do not centre trans people's experiences. Whilst all of these studies engaged with over 1,000 participants, only $14 \%, 3.5 \%$ and $3 \%$ of participants identified as trans respectively. Additionally, the analysis and results presented within these studies tend to focus on the difference in experiences for trans men in comparison to trans women, reinforcing the dominant Western gender binary (Namaste, 2000), overshadowing and neglecting the experiences of those who identify outside or between these imposed categories. Resultantly, the differences between and among trans participants are conflated and the experiences of non-binary, gender-fluid and gender-queer participants are homogenised within a binary understanding of trans identities.

In this paper we address the concerns of participants who represent diverse communities and interrogate the ways in which multiple identity markers interact to create unique experiences of victimisation. As Woods (2014) argues, individuals' experiences of oppression and victimisation are not only shaped by their gender identity, but also by sexuality, race, religion and disability status amongst other things. In complicating the notion of (in)visibility, we add to the extant literature on 'intersectional' difference, and in doing so, we avoid the pitfalls associated with identity politics that have been outlined by Crenshaw (1991) as including the tendency to conflate and overshadow the many differences within a 'community'. 


\section{Methodology}

Empirical data is reported from 31 semi-structured interviews conducted by the first author with trans people who live within the UK and were aged 16 and over. Data was collected and analysed throughout 2018. The interviews formed part of a larger research study that also consisted of an online survey and a discourse analysis of YouTube comments posted on videos relating to 'gender neutral toilets' (Colliver et al., 2019). For the purpose of this paper, we focus on the data elicited from the semi-structured interviews. Semi-structured interviews were conducted to develop a more detailed and contextualised account of trans people's experiences of victimisation. The focus of the interviews was on participants' experiences of hate crime with a specific interest in incidents of verbal abuse, harassment and online victimisation. Participants were asked to share their understanding of hate crime, their potential fear of victimisation and their experiences of hate crime in relation to a number of identity characteristics.

Given the relatively 'hard-to-reach' nature of trans populations, purposive sampling was used to recruit trans people who lived in the UK, were aged 16 and over and who had experienced some form of victimisation targeting their gender identity. Participants were primarily recruited through social media, which although has limitations relating to the representativeness of any given sample, proved to be the most effective method of reaching out to participants. With an 
emphasis on online recruitment, the research was also advertised in a number of community centres, Gender Identity Clinics and charities working with trans people. Significant care was taken in the design of the research project, as not all participants were 18 years old. Given the nature of the research, it was acknowledged that participants may experience emotional and/or psychological distress as a result of retelling their stories. Following ethical approval for the study, participants aged 16 and 17 were recruited without parental/carer consent. It was felt that informing parents/carers could potentially 'out' participants and could result in the participant being at a greater risk of harm than that posed to them by participating. The researcher also had a national network of free youthfriendly support services that participants could fast-track into should they need additional support. The researcher also has significant experience in supporting young LGBTQ people around a number of sensitive, personal issues.

A diverse sample was obtained in relation to gender identity, ethnicity, religion, age and disability status for the semi-structured interviews and was reflective of the broader UK population. When considering the diversity of participants included in the semi-structured interviews, $23 \%$ of interview participants identified as non-binary whilst $31 \%$ identified as male and $44 \%$ identified as female. Participants' ages ranged from 17-67 years old with an average age of 32 years old. In relation to participants' ethnicity, $54.8 \%$ of 
participants identified as White British, making up the majority of participants. However, the rest of the sample was made up of a range of ethnic backgrounds including Black British (10\%), British Asian (3.2\%), Black African and White British (3.2\%), Black Caribbean and White British (3.2\%), White American (3.2\%), South American (3.2\%), Bangladeshi (3.2\%), White European (3.2\%), Irish Traveller (6.4\%), Thai (3.2\%), and Pakistani (3.2\%). Several participants also identified as Christian, Muslim and Sikh. All participants spoke English, although this was to varying levels of fluency. English was the first language for most participants, although four participants spoke English as a second language.

The semi-structured interviews were transcribed verbatim and interviews were fully transcribed. The data was analysed thematically, guided by the six steps outlined by Braun and Clarke (2006). An inductive approach was taken to analyse the data as the lack of current research into the 'everyday' and 'mundane' experiences of hate crime targeting trans people created difficulty in trying to locate pre-existing themes. To engage participants throughout the research process and to gain a greater level of clarity regarding their experiences, participants were invited to review codes and themes developed throughout the analysis of data.

In what follows, we focus on the qualitative data from the interviews to develop two key themes present within participants' narratives: discursively 
constructed visibility and intersectional visibility. To ensure the anonymity of participants, we have assigned pseudonyms in the reporting of findings.

\section{Findings and Discussion}

Whilst the aim of this paper is to present a more complicated reading of the nature of (in)visibility, there was a significant amount of data that speaks to existing research. Therefore, we begin with an overview of the ways in which notions of (in)visibility manifested within dichotomous ways for our participants, before providing a more detailed analysis of the more complicated nature of (in)visibility.

A common thread that underlined participants accounts of victimisation, or indeed, avoidance of victimisation, relate to notions of (in)visibility. An individuals' (in)visibility was key in their understanding of possible victimisation. It was evident within participants' narratives that the fear of victimisation within public spaces was minimal for those who perceived themselves to successfully 'pass' in their gender. In these cases, their perceived 'difference' had been rendered invisible as a result of their ability to conform to physical gendered expectations. On the other hand, individuals who perceived themselves as unable to pass, or for whom passing was not an individual want or need, expressed sentiments of relative feelings of risk of victimisation. When an individual 'passes' in their gender, their trans identity becomes invisible, particularly hidden from the public gaze. 
Participants who perceived themselves as 'passing', and wanted to pass, described the relative ease of accessing sex-segregated spaces, particularly public toilets. Whereas, participants who felt unable to 'pass' expressed significant concern for their safety within these spaces. This is intrinsically linked to the dominant gender binary and expected physical presentations associated with 'male' and 'female' (Namaste, 2000).

Whilst a number of participants constructed the transition process in a fairly linear sense in which the transition from 'non-passing' to 'passing' was clear, it is important to acknowledge that this is not the case for all trans people. 'Passing' was not necessarily considered achievable for all participants and for some, was not necessarily the end goal of their journey. For example, many nonbinary participants did not feel that 'passing' was of any importance, given their identity outside or between categories of 'man' and 'woman'. For some, remaining visibly trans was a personal, or political decision and therefore no active measures were taken to render their 'difference' invisible. This speaks directly to notions of autonomy in relation to identity (Gressgard, 2010) and to the role of the individual in negotiating the ways in which their identities are read.

The notion of 'passing' is widely debated within the literature with claims made that to 'pass' is to live authentically (Sellberg, 2012). Alternatively, to 'pass' has been claimed to conform to, and normalise binary expectations of gender 
presentation (Roen, 2002). Claims made that 'passing' relates to living authentically were evident in this research, particularly in relation to sexsegregated spaces in which participants experienced a higher level of vigilance relating to their gender identity. It can also be seen that 'passing' and becoming 'invisible' to the public gaze may also be employed as a survival technique, to avoid victimisation. Furthermore, it should also be considered that the choice to 'pass' is a privilege that is only available to trans people who are able to 'pass'.

Claims that choosing to 'pass' reinforces normative expectations of gender presentation and choosing not to 'pass' are symbolic of activism and radicalism may be unfounded when it is considered that those who do not 'pass' may not have actively considered this as an option. It may be the case that this is in fact a default position assigned to them as a result of being unable to conform to expected gender presentations. We also complicate notions of 'passing' and claim that to 'pass' is not a static, achieved status. Rather, it is an ongoing process that has different meanings and importance based upon the time and place in which an individual may find themselves. We now present a more detailed analysis of the ways in which (in)visibility goes beyond the apparent dichotomy of invisible and visible.

\section{Discursively Constructed (In)Visibility}


Notions of (in)visibility must also be considered in less tangible ways, to reflect the ongoing negotiation of (in)visibility and the way in which invisibility is not a static, achieved status. For many trans people who experienced hate crime within private spheres such as the home, perpetrated by those closest to them such as family and friends, their victimisation resulted from them 'coming out', or disclosing their trans identity. In this sense, it is not the physical appearance of participants that is the most significantly 'visible' difference. Rather, the 'difference' associated with them is produced in less tangible ways. Therefore, their trans identity is discursively made visible.

'I had to run away when I came out, I brought shame into my family, I embarrassed my family. I was abused and abused by family and who I thought were friends and then I finally had enough and escaped. (Corrina, 21, Female)

'My dad and my brother viciously attacked me, I mean literally beat me to a pulp, I was left with broken ribs, black eyes, a swollen jaw. I mean, they didn't just punch and kick me... I just saw it as something I expected to happen because of my culture and faith and the reactions that I knew 
would come. I was almost prepared for it before I had come out.' (Sam, 31, Male)

In the accounts provided above, Corrina and Sam's 'visibility' as trans is not visually significant. Rather, it is the process of 'coming out' and therefore discursively identifying as trans that renders them 'visible'. For both Corrina and Sam, these incidents occurred before they had begun to publicly present in accordance with their gender. Both were therefore presenting their gender in more 'traditional' ways associated with their sex observed at birth at the time of these incidents. Notions of visibility can therefore be considered complex. Motivations for 'coming out' are many and varied. An individual may feel pressure internally, from a need to live openly, or may feel pressure externally, from family peers and society. However, the process of 'coming out' consequently renders an individuals' 'difference' visible and may lead to subsequent victimisation.

Established literature has demonstrated 'coming out' as an ongoing process, where individuals may continually 'come out' in different social contexts (Zimman, 2009). This was evident in this study, in which participants experienced abuse as a result of continually 'coming out' in a variety of spaces, places and contexts. It can therefore be seen that notions of (in)visibility are fluctuating and continually negotiated in different social settings. Therefore, whilst a dichotomous conceptualisation of (in)visibility may fit within the context of physical appearance, 
it does not appreciate the complexity of (in)visibility. Notions of 'coming out' tie directly in with notions of 'autonomy', and an individual's ability to control when, and in which spaces, their identities are read by others as trans. However, the decision to disclose a trans identity is not always associated with political and activist reasoning. At times, individuals may feel the need to disclose their trans identity due to situational contexts such as physical and sexual relationships. Abuse targeting trans women, more particularly, was often the outcome of the disclosure of their trans identity in these contexts.

‘All of a sudden Kian just jumped up and pushed me. He didn't hit me, but he was holding my neck. He spat in my face before the other guys pulled him off me. Basically, he attacked me because I gave him head and he enjoyed it. Now, he suddenly feels like less of a man, because I am a trans woman. He was so worried about everyone thinking he was gay and his masculinity was challenged.' (Elaine, 48 , Female)

Elaine described the physical violence she experienced as a result of 'coming out' to an individual with whom she had engaged in oral sex with. In this instance, an individual can be simultaneously invisible to the public gaze, but significantly visible within the private sphere. This illustrates the complicated relationship participants have with the concept of 'visibility'. Whilst the most obvious conceptualisation of (in)visibility may appear to be linear, in the sense that an 
individual may transition from 'visible' to 'invisible', the data we have presented evidence a significantly richer understanding of (in)visibility. Furthermore, Elaine's account adds to the complexity of violence against trans women within the context of masculinity and sexuality. The experience described by Elaine demonstrates the material consequences of hetero- and cis-normative social structures, which position trans people as legitimate targets for violence. Notions of 'trans panic' are relevant here. Trans panic may be the result of an internal struggle with sexual identity within a perpetrator as sexual attraction to transgender women challenges hetero- and cis-normative notions of sexuality (Jamel, 2018; Noble, 2012). In this sense, Elaine's experience culminates given the nuanced relationships between invisibility, heteronormativity and cisnormativity. This results in Elaine being responsibilised for her victimisation as a result of 'deception'.

Whilst an individual may 'pass' and their trans identity may therefore not fall under the scrutiny of the public gaze, this does not mean that these individuals do not encounter abuse and hate crime. Previous work regarding 'visibility' has tended to overlook the role of 'visibility' within private spheres, such as the home, in which victimisation may be perpetrated by family, friends and partners. Trans people who 'pass' often experienced abuse and discrimination in these spaces, within the context of familial relationships, friendships and romantic relationships 
when they 'come out' and disclose their trans history, therefore making their 'difference' visible. The idea of 'coming out' is conceptualised as an ongoing process and therefore the perception of risk does not remain static but fluctuates over and throughout time, depending upon the context of any given social situation. We argue that the experiences of victimisation that occur within private spheres can better be accounted for by considering less tangible forms of visibility that have not been addressed by previous research (Pandey, 2004). Therefore, whilst an individual may experience a relative privilege associated with 'passing' in relation to avoiding their gender identity becoming a focus of the 'public gaze', they may find themselves in precarious situations in which their risk of victimisation is increased as a result of the disclosing their trans identity.

\section{Intersectional (In)Visibility}

The concept of '(in)visibility' is a complex, ongoing achievement and individuals may become more, or less 'visibly' trans in different spaces and contexts. It has been noted that a significant amount of research into gender identity is Eurocentric and overwhelmingly White (Jamel, 2018). In exploring trans people's experiences of hate crime, intersectional characteristics proved to be key in our study for understanding how different aspects of identity impact experiences. 
As discussed previously, existing literature has emphasised a visible trans identity with an increased perception in risk of victimisation in public. Yet, at times, the striking visibility of a trans identity is mediated by the 'visibility' of other identity markers.

'Even though I feel like a bigger target because I am a traveller, sometimes it helps, like when people have started abusing me and calling me a tranny and harassing me and then I say something, people shit themselves. Like, there are loads of stereotypes about traveller men, being violent and aggressive, that sometimes you can see people panic as soon as I talk...So, sometimes being a traveller actually makes me feel safer.'

(Emmet, 30, Male)

'I think my appearance has actually helped me avoid being physically attacked. Like, punk aesthetic can be quite intimidating, and I am quite dominating physically, like I'm six foot two, I'm quite big built, and I'm a Queer Punk. Like, that is quite a lot take in. So, yeah, I think in some ways, even though I stand out, sometimes it actually helps keep me safe.'

(Star, 44, Non-Binary) 
In the excerpts above, Emmet and Star both understand the 'difference' they perceive to be visible as a protective factor in their conceptualisation of risk. For Emmet and Star, their feelings of oppression are not necessarily alleviated by a visible difference, but their feelings of the risk of victimisation are eased somewhat, as a result of them being perceived as less 'vulnerable'. In this conceptualisation, Emmet and Star's perception of their visible 'difference' is dissimilar to other participants' perception of their visible difference. It is evidently not the case that if an individual does not 'pass' that victimisation targeting their gender identity is inevitable. As such, we challenge ideas of 'difference' being a static, constant indicator of an individual's 'vulnerability'. Indeed, we argue that stereotypes associated with 'difference' may decrease an individual's perceived vulnerability. The role of stereotypical perceptions of individuals from a traveller culture, characterised by criminality, aggression and physical violence served as a preventative factor in the victimisation of some people. Furthermore, even though some individuals described themselves as 'visibly different' in several ways, physically intimidating presentations of 'difference' also served as a protective barrier to experiencing hate crimes.

This is not to say that those who are characterised and stereotyped as aggressive, physically violent and physically dominant do not face high levels of oppression, discrimination and abuse, as the victimisation of traveller 
communities is well documented (James and Smith, 2017; Wallengren and Mellgren, 2015). Therefore, when managing and negotiating intersecting identities an individual may seek to emphasise an aspect of their identity in order to decrease their risk of victimisation. This demonstrates that there are spatial and structural implications to consider when conceptualising '(in)visibility'. Individuals may seek to draw attention to a particular identity marker when navigating certain spaces. As such, one identity marker functions to compensate against a perceived 'vulnerability' an individual may have due to another intersecting characteristic. However, this relies heavily on the assumption that those reading and engaging with an individual's identity will perceive it in the way intended.

Alternatively, the visibility of a different marginalised characteristic is conceptualised as minimising the risk of experiencing victimisation targeting an individuals' trans identity, but not minimising the risk of victimisation generally. Identities are multiple, fluid and fluctuating, and our self-perceived understanding of identity may not necessarily accord with others' constructions of our identities (Wetherell, 2009). It is therefore key to develop understandings of (in)visibility beyond the current dichotomy. More specifically, research indicates that for trans people, their experiences and presentations of gender are not isolated from other identity markers such as race and class (Stryker, 2006). 
'I do find that I experience more racism than transphobia. I am so visibly Asian and I can't hide that. I can walk with my head down so people can't see my face, but I can't disguise my colour and I think that is what stands out instantly about me, my brownness.' (Sam, 31, Male)

'I think because people can see I'm black and I can't cover that up, people tend to focus on that. I mean don't get me wrong, I usually experience transphobia with racism, but I think it is the colour of my skin that makes people initially notice me...I get anxious that I will be singled out because I look completely different to all of the other black boys on the estate.' (Ty, 21, Non-Binary)

In the excerpts above, participants describe the visibility of their racial identity and an associated increase in their feelings of risk of experiencing abuse. For Sam, the visibility of his Asian heritage becomes the more dominant factor in his accounts of risk. He describes the practical measures he can employ to minimise the visibility of his trans identity but feels unable to take the same precautions to minimise his racial identity. Ty's account also demonstrates a heightened sense of risk as a result of their racial identity, but additionally of failing to meet gendered racial expectations, in which their gender presentation falls outside the realms of what is expected of peers from the same cultural background. Resultantly, Ty experiences a lack of autonomy of the ways in which others perceive their 
identity. As a result, Ty experiences feelings of risk in a broader sense for failing to meet wider societal expectations of gender but is indicative also of a more specific risk in the context of their failure to conform to racial expectations. Consequently, Ty experiences a matrix of oppression, in which multiple identity markers interact and create a unique system of oppression. Understandings of gender and associated gender presentation are heavily racialised and have implications for trans people who may also identify with other marginalised groups.

Not only do intersecting identity markers contribute to the levels of abuse, discrimination and hate crime trans people face, but there are also significant implications for trans people enacting a specific, culturally configured presentation of masculinity or femininity. Culturally and racially configured expectations of masculine and feminine behaviour have implications for the level of (in)visibility trans people are able to achieve. Western culture and therefore cultural expectations of masculinity and femininity are rooted within heteronormative, white-normative ideals (Collins, 2000). Therefore, for trans people who are white, or perceived as white, transition may be seemingly linear in relation to understanding and enacting masculine and feminine expectations. However, for trans people who occupy multiple marginalised identities, there may be cultural, racialised expectations of gender presentation, and therefore 
transition between masculine and feminine presentations and behaviours may be more complex. As a result, trans people may 'pass' or successfully be read as male or female based on their presentation within 'white spaces' but may become more 'visible' within specific contexts in which cultural or racial gendered expectations take precedence. This may be complicated even further in the case of non-binary and gender non-conforming people who may not seek to meet any cultural configuration of gender expectation.

However, it is not just racial identities that were considered by participants to influence their perception of risk. An alternative version of intersectional victimisation is provided by Rose.

'I think people also target me because I am older, I walk with a stick, I'm wrinkly and so people think I'm an easy target, I can't run away, I can't defend myself, so people think I will be easy to abuse. To be fair, they are right, I lose my balance easily, I can't physically defend myself, so I am open to all sorts of abuse.' (Rose, 67, Female)

For Rose, the visibility of her age and physical frailty make her a bigger target for experiencing hate crime. Rose is discussing her experiences of verbal abuse, harassment and physical violence targeting her trans identity. She implies that if she was younger, and less physically frail, she may experience 
less of these incidents. In this sense, her 'visible' vulnerability exacerbates her fear and experiences of victimisation.

Intersectional identity characteristics therefore further complicate notions of '(in)visibility', in which an individual's trans identity is either more or less visible as a result of other visible identity markers. Participants in this study described how other visible characteristics such as race, religion and disability status operated to either overshadow their trans identity or draw attention to their trans identity as a form of secondary victimisation. This adds further complexity to the notion of '(in)visibility', as whilst an individual may perceive themselves as able to 'pass' in relation to their gender identity, they may still experience victimisation based on the visibility of another marginalised identity marker. This may then result in an individual experiencing a matrix of oppression in which their victimisation is motivated by several prejudices.

\section{Conclusion}

Issues of gender identity have become a significant topic of interest within private, political and media spheres and trans communities are gaining considerably more social visibility. In this paper we have presented notions of '(in)visibility' as a complicated and ongoing process and have applied them to individualised accounts of victimisation. Our analysis has allowed for a more nuanced reading 
of the nature of visibility, in which individuals engage with perceptions of risk based on differing levels of 'visibility' associated with them. Existing frameworks within which hate crime victimisation are explored have been discussed and we argue that to understand the experiences of hate crime victims, the role of 'visibility' must also be considered. An individual's level of 'visibility' has implications for which spaces, and by whom they are likely to experience abuse. Managing an individual identity is an intricate, ongoing and fluid process that is achieved both individually and in relation to others. Moreover, we have demonstrated that constructing an individual identity is also culturally and racially situated.

Central to this paper are three key claims. Firstly, in order to better understand how 'hate crime' operates from a criminological perspective, notions of '(in)visibility' should be centred alongside ideas of 'difference' and 'vulnerability'. Existing frameworks within which hate crime is predominantly explored, relating to 'difference' and 'vulnerability' were clearly present in participants' conceptualisation of their experiences. However, the concept of 'visibility' was a consistent finding in this study and we therefore argue that this is a key characteristic that influences an individual's perception of risk of victimisation. Participants in this study often conceptualised their trans identity as 
visibly 'different' and an indication of 'otherness', which in turn, is then conceived to be a significant motivating factor in their victimisation.

Secondly, utilising ideas relating to 'visibility', in particular, acknowledging the role of 'coming out' and less tangible forms of 'visibility' serves as a powerful counter narrative to the dominant focus on 'stranger danger'. This encourages a greater acknowledgement of the significant number of incidents experienced in private spheres, perpetrated by family, friends and colleagues. Failing to recognise the experiences of those deemed 'different' or 'vulnerable' within private spheres contributes to the under reporting of hate crime and can create barriers for victims to recognise their experiences as criminal. Acknowledging various manifestations of '(in)visibility' moves us beyond polarised understandings of 'passing' and its relationship with '(in)visibility'.

Finally, we argue that notions of '(in)visibility' are intrinsically linked to intersecting identity characteristics. In conceptualising '(in)visibility' in more complex ways, we have demonstrated that the relationship between visibility, vulnerability and difference is not simply linear. Within particular contexts, an increased level of visible difference can lead to a perceived reduction in an individual's sense of vulnerability. In this way, existing conceptualisations that associate an increased level of visibility with an inevitable sense of vulnerability do not allow for more granular readings of 'difference'. 
A greater emphasis on the notion of (in)visibility also contributes to current public debate around extending the legislative protection to groups of people who sit outside the currently recognised five monitored strands. Chakraborti and Garland (2012) argue that a vulnerability-based approach allows for greater recognition of marginalised groups who do not currently benefit from legal protection. Exploring 'visibility' in relation to the discursively constructed 'visibility' of difference allows for a greater recognition of those who are already marginalised and may therefore be overlooked within debates of expands hate crime protections ${ }^{2}$. Therefore, when discussing hate crime victimisation in relation to 'difference' and 'vulnerability', we argue that it should be done so alongside a recognition of 'visibility'. We remind readers that visibility is intrinsically linked to individuals' perceptions of the likelihood of them experiencing victimisation or not. It would therefore be problematic to discuss hate crime victimisation without explicitly considering the dichotomous ways in which 'visibility' may exacerbate the likelihood of hate crime victimisation, or alternatively, the way in which 'invisibility' may serve a preventative function to experiencing hate crime victimisation.

\footnotetext{
${ }^{2}$ Groups who could be considered for protection under expanding hate crime protections include individuals with no fixed address and people involved in various aspects of sex work.
} 


\section{References}

Antjoule, N. (2013) 'The Hate Crime Report: Homophobia, Biphobia and Transphobia in London'. London: GALOP.

Ball, M. (2019) 'Unsettling Queer criminology: Notes towards decolonization' Critical Criminology, 27: 145-161.

Ball, M., Buist, C. and Woods, J. (2014) 'Introduction to the special issue on queer/ing criminology: New directions and frameworks' Critical Criminology, 22: 1-4.

Bauer, G. R., R. Hammond, R. Travers, M. Kaay, K.M. Hohenadel and M. Boyce (2009), "I don't think this is theoretical; this is our lives": How erasure impacts health care for transgender people', Journal of the Association of Nurses in AIDS Care, 20 (5), 348-361.

Braun, V. and Clarke, V. (2006) 'Using thematic analysis in psychology' Qualitative Research in Psychology, 3(2): 77-101.

Browne, K. (2004) Genderism and the bathroom problem: (Re)materialising sexed sites, (re)creating sexed bodies, Gender, Place and Culture, 
11(3), 331-346.

Burgess, A., Regehr, C. and Roberts, A. (2013) 'Victimology: Theories and Applications' (2nd Edition) Burlington: Jones and Bartlett Learning.

Chakraborti, N. and Garland, J. (2012) 'Reconceptualizing hate crime victimization through the lens of vulnerability and 'difference' Theoretical Criminology, 16(4): 499-514.

Chakraborti, N., \& Zempi, I. (2012) The veil under attack: Gendered dimensions of Islamophobic victimization International Review of Victimology, 18(3): 269-284.

Chakraborti, N., Garland, J. and Hardy, S. (2014) 'The Leicester Hate Crime Project: Findings and Conclusions' Leicester: University of Leicester. Collins, P. (2000) Black Feminist thought: Knowledge, consciousness, and the politics of empowerment. ( $\left.2^{\text {nd }} \mathrm{Ed}\right)$ New York: Routledge.

Colliver, B., Coyle, A. and Silvestri, M. (2019) The 'online othering' of transgender people in relation to 'gender neutral toilets', In: Lumsden, K. and Harper, E. (eds) Online Othering: Exploring Digital Violence and 
Discrimination on the Web, London: Palgrave Macmillan, 215-238.

Crenshaw, K. (1991) 'Mapping the margins: Intersectionality, identity politics, and violence against women of color' Stanford Law Review, 43(6): 1241-1299.

Davidoff, L. (2003) Gender and the "Great Divide": Public and private in British gender history, Journal of Women's History, 15(1), 11-27.

Doan, P. (2010). The tyranny of gendered spaces: Reflections from beyond the gender dichotomy, Gender, Place \& Culture, 17, 635-654.

Gerstenfeld, P. (2004) 'Hate Crimes: Causes, Controls and Controversies'. London: Sage Publications.

Gressgård, R. (2010) When trans translates into tolerance - or was it monstrous? Transsexual and transgender identity in liberal humanist discourse, Sexualities, 13(5), 539-561

Hall, N. (2005) ‘Hate Crime’. Cullompton: Willan Publishing.

Hines, S. (2010) 'Introduction'. In: Hines, S. and Sanger, T. (eds) Transgender Identities: Towards a Social Analysis of Gender Diversity, New York: Routledge. 1-22. 
Home Office. (2012) 'Challenge It, Report It, Stop It: The Governments Plan to Tackle Hate Crime', London: Home Office.

Home Office. (2019) 'Hate Crime: England and Wales, 2018/19'. London: Home Office.

Jacobs, J. and Potter, K. (1998) 'Hate Crimes: Criminal Law and Identity Politics'. Oxford: Oxford University Press.

Jamel, J. (2018) 'Transphobic Hate Crime'. London: Palgrave Macmillan.

James, S. E., Herman, J. L., Rankin, S., Keisling, M., Mottet, L., \& Anafi, M. (2016). The Report of the 2015 U.S. Transgender Survey. Washington, DC: National Center for Transgender Equality.

James, Z., \& Smith, D. (2017). 'Roma inclusion post Brexit: A challenge to existing rhetoric?' Safer Communities, 16(4): 186-195.

Jauk, D. (2013) ‘Gender violence revisited: Lessons from violent victimization of transgender identified individuals' Sexualities, 16(7): 807-825.

Johnson, K., Faulkner, P., Jones, H. and Welsh, E. (2007) 'Understanding Suicide and Promoting Survival in LGBT Communities'. Brighton: 
University of Brighton.

Kidd, J. and Witten, T. (2008) 'Transgender and transsexual identities: The next strange fruit-hate crimes, violence and genocide against the global transcommunities' Journal of Hate Studies, 6(1): 31-63.

Kilian, E. (2014) 'Claiming space: Transgender visibility in the arts' In: Zabus, C. and Coad, D. (eds.). Transgender Experience: Place, Ethnicity, and Visibility. New York: Routledge. 85-107.

Lawrence, F. (1999) 'Punishing Hate: Bias Crimes Under American Law'. Cambridge: Harvard University Press.

Lovelock, M. (2017) 'Acceptance, Humanity and Emotional Excess: The Politics of Queer Suffering in Big Brother UK' European Journal of Cultural Studies, 20(4): 449-63.

Mason-Bish, H. (2010) 'Future challenges for hate crime policy: Lessons from the past'. In: Chakraborti, N. Hate Crime: Concepts, Policy, Future Directions. (eds). Cullompton: Willan. Pp. 58-77.

Mattias de Vries, K. (2012) 'Intersectional identities and conceptions of the self: 
The experience of transgender people' Symbolic Interaction, 35(1): 49-67.

McGhee, D. (2007) 'The challenge of working with racially motivated offenders:

An exercise in ambivalence?' Probation Journal, 54(3): 213-226.

Meyer, D. (2014) 'Resisting Hate Crime Discourse: Queer and Intersectional

Challenges to Neoliberal Hate Crime Laws'. Critical Criminology. 22. Pp. 113- 125.

Mills, C. (2019) 'Gay visibility and disorganized and strained communities: A community-level analysis of anti-gay hate crime in New York City' Journal of Interpersonal Violence, 1-22.

METRO. (2014) 'Youth Chances: Summary of First Findings'. London: METRO.

Monro, S. (2003) 'Transgender politics in the UK' Critical Social Policy, 23(4): 433-452.

Namaste, V. (2000) Invisible Lives: The Erasure of Transsexual and Transgender People, Chicago: University of Chicago Press.

Noble, B. (2012) Trans. Panic. Some thoughts toward a theory of feminist, In: Enke, A. (eds) Transfeminist Perspectives in and Beyond Transgender 
and Gender Studies, Philadelphia: Temple University Press, 45-59.

Pandey, A. (2004) 'Constructing Otherness: A Linguistic Analysis of the Politics of Representation and Exclusion in Freshmen Writing' Issues in Applied Linguistics, 14(2): 153-184.

Panfil, V. (2017) 'The Gang's all Queer: The Lives of Gay Gang Members', New York: New York University Press.

Perry, B. (2001) 'In the Name of Hate: Understanding Hate Crimes', London: Routledge.

Perry, B. (2015) 'All of a Sudden, There Are Muslims': Visibilities and Islamophobic Violence in Canada' International Journal for Crime, Justice and Social Democracy, 4(3): 4-15.

Perry, B. and Dyck, D. (2014) “I Don't Know Where It Is Safe”: Trans Women's Experiences of Violence' Critical Criminology, 22(1): 49-63.

Roen, K. (2002) "Either/Or" and "Both/Neither": Discursive tensions in transgender politics' Signs, 27(2): 501.522.

Rundall, E. and Vecchietti, V. (2010) '(In)Visibility in the workplace: The 
experiences of trans employees in the UK' In: Hines, S. and Sanger, T. (eds). Transgender Identities: Towards a Social Analysis of Gender Diversity, New York: Routledge. 127-152.

Sellberg, K. (2012) 'Transgender identity and passing authentically. Pro-passing transgender identity and literature: (post-)transsexual politics and poetics of passing' In: Cooley, D. and Harrison, K. (eds). Surrey, England: Ashgate Publishing. 211-219.

Serano, J. (2007) 'Whipping Girl: A Transsexual Woman on Sexism and the Scapegoating of Femininity', California: Seal Press.

Spalek, B. (2008) Communities, Identities and Crime, Bristol: Policy Press.

Stotzer, R. (2009) Violence against transgender people: A review of the United States data, Aggression and Violent Behaviour, 14, 170-179.

Stryker, S. (2006) '(De)Subjugated knowledges: An introduction to transgender studies' In: Stryker, S. and Whittle, S. Transgender Studies Reader, New York: Routledge. 1-18.

Turner, L., Whittle, S., and Combs, R. (2009) 'Transphobic Hate Crime in the 
European Union'. London: Press for Change.

Wallengren, S. and Mellgren, C. (2015) ‘The role of visibility for a minority's exposure to (hate) crime and worry about crime - a study of the Traveller community' International Review of Victimology, 21(3): 303-319.

Walters, M. (2011) 'A general theories of hate crime? Strain, doing difference and self-control'. Critical Criminology, 19, 313-330.

Wetherell, M. (2009) Theorizing Identities and Social Action, London: Palgrave Macmillan.

Woods, J. (2014) 'Queer contestations and the future of a critical "queer” criminology' Critical Criminology, 22(1): 5-19.

Zimman, K. (2009) 'The other kind of coming out': Transgender people and the coming out narrative genre, Gender and Language, 3, 53-80. 